\title{
Keine Wirkung beim akuten Schlaganfall
}

\section{Hier steht eine Anzeige.}

Fragestellung: Ist eine transkranielle Lasertherapie bei Patienten mit akutem ischämischem Insult wirksam?

Hintergrund: Die transkranielle Lasertherapie benutzt Laseremissionen im Nah-Infrarotbereich. Sie war in Tierexperimenten neuroprotektiv wirksam und in einer Phase-II-Studie mit $660 \mathrm{~Pa}-$ tienten ergab sich ein positiver Trend [1].

Patienten und Methodik: Für die Phase-III-Studie NEST 3 war der Einschluss von 1.000 Patienten geplant, die innerhalb von 24 Stunden entweder mit der transkraniellen Lasertherapie oder mit einer Scheintherapie behandelt werden sollten.

Ergebnisse: Die Studie wurde vom Data Monitoring Committee abgebrochen, nachdem Ergebnisse von 566 Patienten vorlagen. Die Interimsanalyse legte nahe, dass auch bei einer höheren Patientenzahl kein thera-

Hacke W, Schellinger PD, Albers GW et al; NEST 3 Committees and Investigators.

Transcranial laser therapy in acute stroke treatment: results of neurothera effectiveness and safety trial 3 , a phase III clinical end point device trial. Stroke 2014; 45: 3187-93 peutischer Effekt der transkraniellen Lasertherapie zu erwarten ist.

Schlussfolgerungen: Die transkranielle Lasertherapie ist bei Patienten mit akutem ischämischem Insult nicht wirksam.

\section{- Kommentar von Hans-Christoph Diener, Essen}

\section{Ungesicherte Studienfinanzierung}

Die NEST-3-Studie wird hier nicht vorgestellt, um eine weitere negative Studie zur neuroprotektiven Therapie zu referieren, sondern weil sie zeigt, wie problematisch es ist, wenn große Studien mit Wagniskapital durchgeführt werden. Die Firma, die diese Studie finanzierte, meldete wenige Tage nach dem Bekanntwerden des negativen Ergebnisses Insolvenz an und entließ schlagartig alle Mitarbeiter. Die Studienzentren blieben zum Großteil auf nicht bezahlten Vergütungen sitzen und mussten sich darum kümmern, die Lasertherapiegeräte fachgerecht zu entsorgen. Für das Steering-Committee war es extrem problematisch, die Daten zu erhalten und zu publizieren. Bei zukünftigen Studien, die über Wagniskapitalgeber finanziert werden, muss a priori eine gewisse Summe auf ein Treuhandkonto platziert werden, um Firmenangestellte so lange zu bezahlen, bis die Studie angemessen beendet und ausgewertet ist. 